\title{
Traditional Korean Medicine as Collaborating Treatments with Conventional Treatments for Knee Osteoarthritis: A Protocol for a Systematic Review and Meta-Analysis
}

\author{
Yeon-Cheol Park (iD) ${ }^{1, *}$ \\ Bon-Hyuk Goo iD ${ }^{2, *}$ \\ Kyeong-Ju Park iD ${ }^{3}$ \\ Jun-Yeon Kim (iD ${ }^{3}$ \\ Yong-Hyeon Baek (iD) \\ 'Department of Acupuncture \& \\ Moxibustion, College of Korean \\ Medicine, Kyung Hee University, Seoul, \\ 02447, South Korea; ${ }^{2}$ Department of \\ Acupuncture \& Moxibustion, Kyung Hee \\ University Hospital at Gangdong, Seoul, \\ 05278, South Korea; ${ }^{3}$ Department of \\ Clinical Korean Medicine, Graduate \\ School, Kyung Hee University, Seoul, \\ 02447, South Korea \\ *These authors contributed equally to \\ this work
}

Background: Knee osteoarthritis (OA) is a degenerative disease of the joint cartilage with no definite treatment in the early stage. Several previous review studies have shown that alternative medical treatments, including acupuncture, moxibustion, and herbal medicines, are effective in improving the symptoms of the disease and the patient's quality of life. However, no systematic review study has shown the effectiveness of the combination of conventional and alternative therapies. Therefore, the aim of our study is to determine the most effective combination therapies and to provide evidence for the effectiveness and safety of integrated therapies. This article describes the protocol for the methods that will be applied in our systematic review.

Methods: We will conduct an electronic search of nine databases: PubMed, Embase, Cochrane, Google Scholar (first 100 articles), four Korean databases (KoreaMed, Korean Studies Information Service System, Research Information Service System, and Oriental Medicine Advanced Searching Integrated System), and one Chinese database (China National Knowledge Infrastructure). Only randomized controlled studies that reported on both conventional treatment (drugs and hyaluronic acid) and traditional Korean medicine (acupuncture, moxibustion, and herbal medicines) will be selected. The primary outcomes will be pain and function of the joint. The secondary outcomes will include pain relief duration, total effective rate, incidence of adverse events, and quality of life. We will assess the methodological quality of the included studies using the Cochrane risk of bias tool. For the meta-analysis, standardized mean differences and risk ratios with $95 \%$ confidence intervals will be applied for continuous and dichotomous data, respectively.

Results: This review will evaluate the effectiveness and safety of several Korean medicine treatments combined with conventional treatments for knee OA.

Conclusion: Our review will provide a good foundation for the integrative treatment of knee OA. Keywords: acupuncture, herbal medicine, hyaluronic acid injection, knee osteoarthritis, moxibustion, NSAIDs

\section{Introduction}

Knee osteoarthritis (OA) is a major disease that accounts for approximately $81.8 \%$ of all diseases associated with degeneration of the ligament and cartilage surrounding the knee joint. ${ }^{1}$ Conventional treatments for knee arthritis, such as nonsteroidal anti-inflammatory drugs (NSAIDs) and hyaluronic acid (HA) injection, have been recommended for patients with knee OA. ${ }^{2}$ However, these treatments are limited to
Correspondence: Yong-Hyeon Baek Department of Acupuncture \&

Moxibustion, College of Korean Medicine, Kyung Hee University, 26, Kyungheedae-ro, Dongdaemun-gu, Seoul, 02447, South Korea

Tel +82024408642

Fax +82024407770

Email byhacu@khu.ac.kr 
pain relief and none of them can reverse joint cartilage damage or prevent the progression of arthritis. Despite the varied accessibility of conventional treatments, no single therapy is unequivocally successful. Therefore, when nonsurgical treatment is needed (Kellgren and Lawrence [KL] grade 2-3), effective integrative treatment is required to relieve pain and prevent disease exacerbation. ${ }^{3}$

Various alternative medical treatments are available for improving knee pain or function, and various kinds of acupuncture, moxibustion, and herbal medicine have been used for a long time in traditional Korean and Chinese medicine, individually or in combination. Many studies have shown the efficacy of various kinds of acupuncture, moxibustion, and herbal medicine in relieving knee OA in terms of attenuating disease symptoms, improving quality of life and knee function, and reducing the frequency, duration, and severity of exacerbations. ${ }^{4-6}$ Clinical trials have reported that various kinds of acupuncture, such as manual acupuncture, electroacupuncture, and pharmacoacupuncture, are able to relieve knee pain. ${ }^{7}$ Considering that moxibustion is an adjunct therapy to acupuncture, the effect of moxibustion as a treatment for $\mathrm{OA}$ is also clinically relevant. ${ }^{8}$ Herbal medicine or herbal products have been used and recommended by many clinicians. They have been shown to alleviate the symptoms of knee $\mathrm{OA}$ and to reduce costs. ${ }^{9}$

According to studies on the mechanisms of moxibustion and acupuncture, these treatments relieve chronic inflammatory pain by inhibiting the production of inflammatory mediators such as interleukin- $\beta$, prostaglandin E2, and cyclooxygenase-2. ${ }^{10}$ Electroacupuncture exerts curative effects against knee OA through several mechanisms, such as by decreasing the expression of Bax and caspase- 3 proteins in the rectus femoris muscle, increasing the ratio of $\mathrm{Bcl}-2 / \mathrm{Bax}$ proteins, and promoting pathological changes in cartilage cells. ${ }^{11}$ Bee venom contains several components that exhibit pharmacological activities, including anti-inflammatory, anti-nociceptive, and antiarthritic effects, through the inhibition of the DNAbinding activity of nuclear factor- $\mathrm{\kappa B}$ and prostaglandin synthesis through cyclooxygenase inhibition. ${ }^{12}$ Herbal medicines used in knee OA have been shown to be effective in enhancing the chondrogenic differentiation of CD105 and type II collagen, inhibiting sodium nitroprusside-induced chondrocyte apoptosis, and regulating the expression of vascular endothelial growth factor and hypoxia-inducible factor- $1 \alpha .{ }^{13}$ A recent review indicated that acupuncture, moxibustion, and herbal medicine have important analgesic effects and are safe to use, in contrast to drugs that have adverse effects, for the treatment of knee OA. Meanwhile, many randomized controlled trials (RCTs) have demonstrated the efficacy and safety of adjunct therapy for knee OA. Such studies have shown that acupuncture, moxibustion, and herbal medicine could be potential combination treatment candidates with antiinflammatory and cartilage-protective effects through the enhancement of chondrogenic differentiation. ${ }^{14,15}$

Clinical studies have also found that alternative medical treatments could be feasible therapeutic options for knee OA. Alone or in combination with conventional pharmaceutical drugs, alternative medical treatments have also been commonly used for the clinical management of knee OA. Some systematic reviews have demonstrated the efficacy and safety of acupuncture, moxibustion, and herbal medicine in the treatment of knee OA. However, most of the systematic reviews were based on interventions related to various traditional medicines, and there is no systematic review comparing the effectiveness of combination therapy and standard treatment. ${ }^{16-18}$ To assist clinical practice and potentially reduce the burden of knee OA, it is important to systematically review the current evidence on integrative treatments compared with conventional treatments.

With this background, the objective of our study is to provide descriptive summaries of the baseline characteristics, various treatment methods, and number of therapy sessions in patients with knee arthritis for whom integrative treatment is applicable. Another purpose of the study is to compare the clinical applicability of integrative treatment involving various alternative medical therapies. Therefore, we will conduct a systematic review and meta-analysis of RCTs to compare conventional treatments (NSAIDs and HA) versus alternative medical treatments and integrative treatment versus conventional treatment alone.

\section{Methods \\ Study Registration}

This systematic review has been registered at Research Registry (registration no. reviewregistry1086, http://www. researchregistry.com).

\section{Eligibility Criteria}

Types of Studies

Only RCTs published in English, Korean, and Chinese will be included to evaluate the effect of integrative treatment 
in patients with knee OA. Non-RCTs, quasi-RCTs, animal studies, case series, case reports, uncontrolled trials, and laboratory studies will be excluded.

\section{Types of Patients}

Patients diagnosed with knee OA will be included in the study. No limitations will be imposed on disease severity, age, sex, race, and education status of the patients. Patients will be classified as having knee OA using either of the following criteria:

1. Grade 2 or more according to the KL classification system $^{19}$

2. Meeting the American College of Rheumatology criteria for knee OA. ${ }^{20}$

\section{Types of Interventions}

Studies in which groups were treated using conventional treatments such as NSAIDs and HA injections will be included. RCTs that compared conventional treatments to other adjunct therapies, such as acupuncture, herbal medicine, and moxibustion, will also be considered eligible. Acupuncture as an experimental intervention is defined as the penetrating stimulation of acupoints by using needles. Various kinds of acupuncture, such as manual acupuncture, electroacupuncture, and phamacoacupuncture, will be included. Studies that used any type of moxibustion (direct or indirect) for treating OA will be included. Herbal medicine for treating knee $\mathrm{OA}$ based on syndrome differentiation, according to the traditional medical theory, will also be included. Studies on new prescriptions not listed in the 10 herbal medicine books recommended by the Korean Food and Drug Administration or herbal products derived from experiments will be excluded.

In summary, the following comparisons will be addressed:

1. Conventional treatment (NSAIDs and HA) versus alternative medical treatments

2. Integrative treatment versus conventional treatment alone.

\section{Types of Outcome Measures Primary Outcomes}

Primary outcomes concerning joint pain and function will be assessed using the visual analog scale or numerical rating scale, Western Ontario and McMaster Universities Arthritis Index (total Western Ontario and McMaster
Universities Arthritis Index scores), Lequesne functional index (total Lequesne scores), and Lysholm knee score scale (total Lysholm scores).

\section{Secondary Outcomes}

The secondary outcomes will include the following:

1. Total effective rate (pain relief rate)

2. Incidence of adverse events

3. Quality of life

\section{Information Sources and Search Strategy}

A literature search will be performed using the following electronic databases: PubMed, Embase, Cochrane, Google Scholar (first 100 articles), four Korean medical databases (KoreaMed, Korean Studies Information Service System, Research Information Service System, and Oriental Medicine Advanced Searching Integrated System), and one Chinese database (China National Knowledge Infrastructure). A secondary search will include the reference lists of pertinent articles and relevant systematic reviews. The databases will be searched for articles published from January 1, 2000, to January 1, 2021. The search terms will be a combination of key words related to diagnosis and treatment. We will focus on MeSH (Medical Subject Headings) terms such as "knee osteoarthritis" along with text searches of terms such as "knee osteoarthritis," "acupuncture," "electroacupuncture," "pharmacoacupuncture," "bee venom," "moxibustion, "herbal medicine," "traditional Chinese medicines," or "herbal extract." A search for each study method will be conducted. The search strategy for PubMed is shown in Table 1.

\section{Data Collection and Analysis Selection Process}

We will upload the search results into a systematic review software RevMan 5.4 (The Cochrane Collaboration, London, UK). Firstly, any duplicate studies will be excluded. In the first round of screening, two reviewers (YP and KP) will independently screen the titles and abstracts of the retrieved articles to exclude any obviously irrelevant articles. After the abstract screening, eligibility will be assessed through full-text screening. Eligibility at both levels (abstract and full text) will be assessed independently and in duplicate to maintain objectivity and transparency. The suitability for inclusion of the full texts of the remaining articles will be judged based on the 
Table I PubMed Search Strategy

\begin{tabular}{|c|c|}
\hline$\# 1$ & Knee osteoarthritis [MeSH] \\
\hline$\# 2$ & Knee osteoarthritides $[\mathrm{MeSH}]$ \\
\hline$\# 3$ & Knee osteoarthritis [title/abstract] \\
\hline$\# 4$ & Knee osteoarthritides [title/abstract] \\
\hline$\# 5$ & Knee [title/abstract] \\
\hline \#6 & Knee joint [title/abstract] \\
\hline$\# 7$ & Degenerative arthritis [title/abstract] \\
\hline \#8 & $\# I$ OR \#2 OR \#3 OR \#4 OR \#5 OR \#6 OR \#7 \\
\hline \#9 & Acupuncture [title/abstract] \\
\hline$\# 10$ & Eletroacupuncture [title/abstract] \\
\hline$\# 11$ & Electro-acupuncture [title/abstract] \\
\hline$\# 12$ & Acutherapy [title/abstract] \\
\hline$\# 13$ & Acupuncture therapy [title/abstract] \\
\hline$\# 14$ & Acupuncture therapies [title/abstract] \\
\hline$\# 15$ & Acupuncture treatment [title/abstract] \\
\hline$\# 16$ & Acupuncture treatments [title/abstract] \\
\hline$\# 17$ & Acupoint [title/abstract] \\
\hline$\# 18$ & Acupressure [title/abstract] \\
\hline$\# 19$ & $\begin{array}{l}\# 9 \text { OR \#I0 OR \#II OR \#I2 OR \#I3 OR \#I4 OR \#I5 OR } \\
\# 16 \text { OR \#I7 OR \#I8 }\end{array}$ \\
\hline$\# 20$ & Pharmacoacupuncture [title/abstract] \\
\hline$\# 21$ & Hydroacupuncture [title/abstract] \\
\hline$\# 22$ & Bee [title/abstract] \\
\hline$\# 23$ & Bee venom [title/abstract] \\
\hline$\# 24$ & Bee sting [title/abstract] \\
\hline$\# 25$ & Acupoint injection [title/abstract] \\
\hline$\# 26$ & Apitoxin [title/abstract] \\
\hline$\# 27$ & Apitherapy [title/abstract] \\
\hline \#28 & $\begin{array}{l}\# 20 \text { OR \#2I OR \#22 OR \#23 OR \#24 OR \#25 OR \#26 OR } \\
\# 27\end{array}$ \\
\hline$\# 29$ & Moxibustion [title/abstract] \\
\hline$\# 30$ & Moxa [title/abstract] \\
\hline$\# 31$ & Moxibustion treatment [title/abstract] \\
\hline \#32 & Moxibustion treatments [title/abstract] \\
\hline \#33 & $\# 29$ OR \#30 OR \#31 OR \#32 \\
\hline
\end{tabular}

(Continued)
Table I (Continued).

\begin{tabular}{|l|l|}
\hline$\# 34$ & Chinese medications [title/abstract] \\
\hline$\# 35$ & Chinese medicines [title/abstract] \\
\hline$\# 36$ & Herbal medicine [title/abstract] \\
\hline$\# 37$ & Decoction [title/abstract] \\
\hline$\# 38$ & Granule [title/abstract] \\
\hline$\# 39$ & Korean traditional medicine [title/abstract] \\
\hline$\# 40$ & Extract [title/abstract] \\
\hline$\# 41$ & Herbal [title/abstract] \\
\hline$\# 42$ & $\# 34$ OR \#35 OR \#36 OR \#37 OR \#38 OR \#39 OR \#40 OR \\
\hline$\# 41$ \\
\hline$\# 43$ & Non-surgical [title/abstract] \\
\hline$\# 4$ & Non-steroidal anti-inflammatory agent [title/abstract] \\
\hline$\# 45$ & Viscosupplementation [title/abstract] \\
\hline$\# 46$ & $\# 43$ OR \#44 OR \#45 \\
\hline$\# 47$ & $\# 8$ AND \#19 \\
\hline$\# 48$ & $\# 8$ AND \#28 \\
\hline$\# 49$ & $\# 8$ AND \#33 \\
\hline$\# 50$ & $\# 8$ AND \#42 \\
\hline$\# 5 I$ & $\# 8$ AND \#46 \\
\hline
\end{tabular}

eligibility criteria. Then we will cross-check the opinions of two reviewers. If the two reviewers will not be able to reach an agreement, a third reviewer (YHB) will make the final decision. (Figure 1)

\section{Data Management and Extraction}

The studies will be managed within the EndNoteX9 reference management system. Data extractors, working independently, will collect primary data from the included trials using RevMan software. Two reviewers (YCP and KJP) will independently extract data from the selected studies and complete the data collection form for each. Any discrepancies or uncertainties will be resolved through a discussion with a senior reviewer (YHB). If the reported data in the studies are unclear, missing, or presented in a form that is either unextractable or difficult to reliably extract, the study authors will be contacted for clarification. All data will be cross-checked and transferred to RevMan software. The following data will be extracted: 


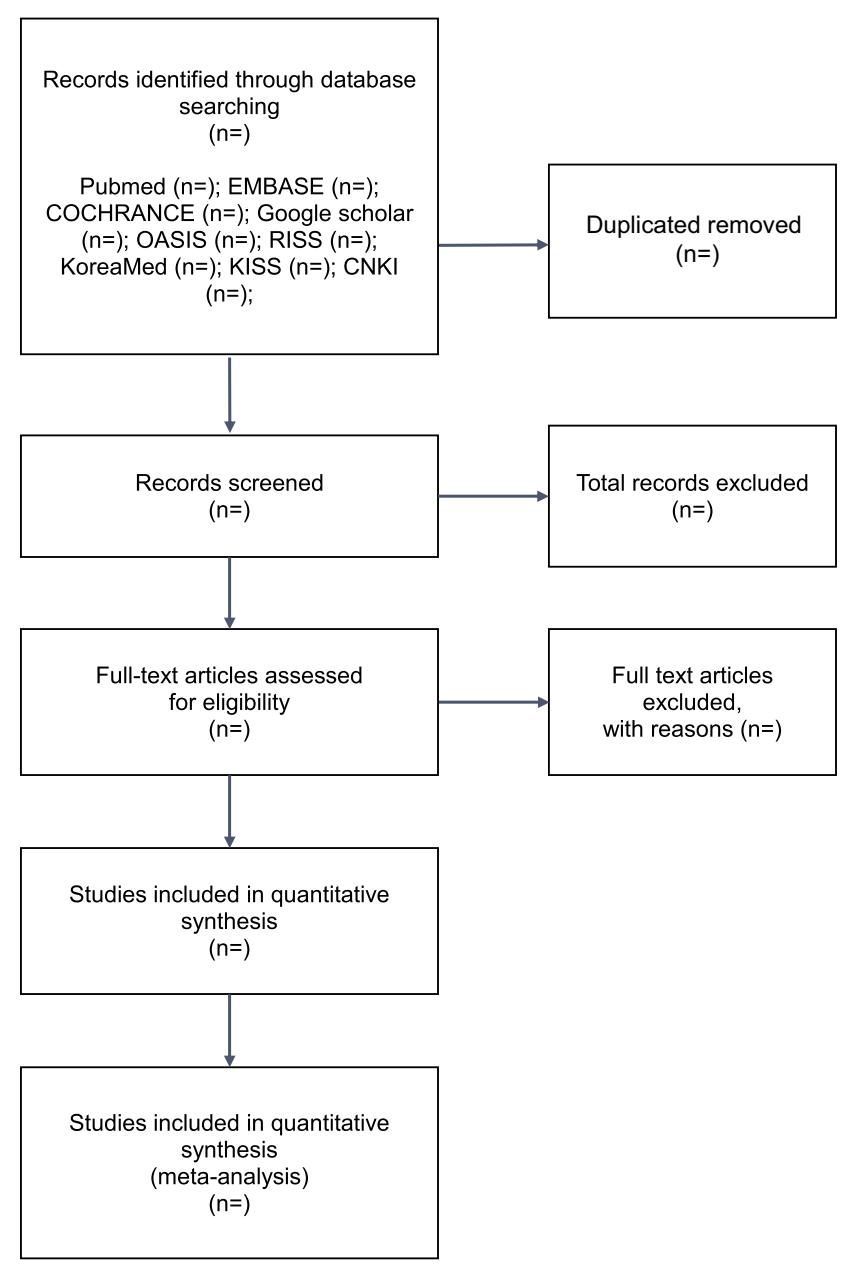

Figure I Flow diagram of selection process.

Notes: Adapted from Page MJ, McKenzie JE, Bossuyt PM, Boutron I, Hoffmann TC, Mulrow CD, et al. The PRISMA 2020 statement: an updated guideline for reporting systematic reviews. BMJ 2021;372:n7I. ${ }^{24}$

1. General information: research identifier, publication year, article title, first author, corresponding author, contact information, journal name, and country.

2. Study methods: study design, sample size, randomization method, allocation concealment, blinding, incomplete or selective reporting, and other sources of bias.

3. Participants: inclusion and exclusion criteria, age, sex, race, onset, and diagnostic criteria for knee OA.

4. Interventions: conventional intervention (drug or HA)/type of adjunct therapy, treatment details, treatment duration, and treatment frequency.

5. Outcomes: primary and secondary outcomes (as described above).

\section{Risk of Bias in Individual Studies}

We will use the Cochrane Collaboration risk of bias tool to evaluate the methodological quality of the included studies. The risk of bias in the included studies will be independently assessed by two reviewers (YCP and KJP). The following items will be assessed: random sequence generation, allocation concealment, blinding of participants and personnel, blinding of outcome assessment, incomplete outcome data, selective outcome reporting, losses to follow-up, intention-to-treat analysis, handling of missing data, funding sources, and other biases. Each item will be assigned a low, unclear, or high risk of bias, and any discrepancies will be resolved through a discussion between the two reviewers or by consulting a senior reviewer (YHB).

\section{Assessment of Heterogeneity}

Heterogeneity will be assessed by visually inspecting the forest plots to detect nonoverlapping confidence intervals (CIs) and by investigating the $\chi 2$ ( $\mathrm{P}>0.10$ will be considered to indicate no heterogeneity) and 12 statistics. An I 2 value of $\geq 75 \%$ will be considered to indicate considerable heterogeneity. If considerable heterogeneity will be identified, the possible causes will be explored through subgroup analyses.

\section{Data Synthesis}

We will use RevMan 5.4 (The Cochrane Collaboration, London, UK) to perform the meta-analysis. For continuous data, the results will be presented as standardized mean differences with 95\% CIs. For dichotomous outcomes, data will be expressed as risk ratios with 95\% CIs. A random-effect or fixed-effect model will be used to calculate the pooled estimates of the effect size. If we will not be able to conduct a meta-analysis because of a lack of clinical studies or because of heterogeneity, we will present the effect size and $95 \%$ CI of every outcome in each clinical trial and describe the significance of important results in the results section. To summarize the findings of the meta-analysis and describe the strength of evidence, we will use the Grades of Recommendation, Assessment, Development, and Evaluation approach.

\section{Subgroup Analyses}

We plan to perform subgroup analyses if substantial heterogeneity will be identified. Subgroup analyses will be conducted according to the following items: 
1. Type of conventional treatment (NSAIDS and hyaluronic acid injection)

2. Type of acupuncture

3. Type of used NSAIDs

4. Degree of severity

We plan to classify according to the above criteria and perform meta-analysis to find out if heterogeneity is resolved within the group. If there is no heterogeneity within the group, it can be regarded that there is no inconsistency when judging the quality of evidence.

\section{Assessment of Reporting Biases}

If the number of included studies will be $>10$, we will generate funnel plots to detect reporting biases and smallstudy effects.

\section{Discussion}

Degenerative arthritis is the most preferred disease to study when considering medical needs, such as the number of patients to be treated and the total medical cost. The number of patients treated for knee OA in Korea was approximately 3.68 million in 2016, and the average annual increase rate during the last 5 years was $3.2 \%$. In particular, knee arthritis, which accounts for $81.8 \%$ of all degenerative arthritic diseases, is a disease that requires intensive management. ${ }^{21}$ The treatment of early knee OA is limited to pain relief, and no treatment can reverse or prevent the progression of joint cartilage damage. ${ }^{22}$ For this reason, there is a continuing need to combine traditional Korean medicine treatment with standard treatment in the nonsurgical stage.

The Korea Health Industry Development Institute planned a project on the research progress and development of integrated treatment guidelines, including traditional Korean medicine treatments or other interventions such as exercise and diet. Six major diseases for which combination therapies are effective were selected through a review of medical field requirements and the results of clinical studies. We are developing a clinical pathway for combination therapy for knee arthritis.

To develop an integrative clinical pathway for knee $\mathrm{OA}$, the clinical practice guideline for knee OA was searched and an analysis of major interventions was completed. The efficacy and safety of traditional Korean medicine treatments such as acupuncture, moxibustion, and herbal medicine for knee OA have been reported in various clinical studies and systematic reviews. However, in terms of the standard treatments recommended by the clinical practice guideline, systematic analysis studies on their combination with traditional Korean medicine treatments are lacking.

The purpose of our study is to identify the most effective treatment for knee OA when combined with standard treatments such as NSAIDs and HA injection. The search period of the study will be extended to 2020 to include the most recent studies. To provide compelling evidence and better guidance in clinical practice, all steps in this review will be performed according to Cochrane Handbook 5.2.0..$^{23}$

Our review will provide current clinical evidence on the effectiveness and safety of integrative treatments for knee OA. This review may also be helpful when designing further clinical studies on knee OA. The results will provide useful information to practitioners and patients, and will be helpful in setting guidelines for other countries that may not be familiar with combination therapies involving traditional Korean medicine. Ultimately, it can provide the basis for medical policy decisions for the integrative treatment of knee OA.

\section{Abbreviations}

OA, osteoarthritis; NSAIDs, non-steroidal antiinflammatory drugs; HA, hyaluronic acid; AT, acupuncture; RCT, randomized controlled trial; KL, Kellgren and Lawrence classification system; CI, confidence interval.

\section{Author Contributions}

All authors made substantial contributions to conception and design, acquisition of data, or analysis and interpretation of data; took part in drafting the article or revising it critically for important intellectual content; agreed to submit to the current journal; gave final approval of the version to be published; and agree to be accountable for all aspects of the work.

\section{Funding}

This research is supported by a grant from the Korea Health Technology R\&D Project through the Korea Health Industry Development Institute, funded by the Ministry of Health \& Welfare, Republic of Korea (grant no. HI20C1405). 


\section{Disclosure}

Yeon-Cheol Park and Bon-Hyuk Goo are co-first authors for this study. All authors have no conflicts of interest to disclose.

\section{References}

1. Kan HS, Chan PK, Chiu KY, et al. Non-surgical treatment of knee osteoarthritis. Hong Kong Med J. 2019;25(2):127-133. doi:10.12809/ hkmj 187600

2. Miller LE, Fredericson M, Altman RD. Hyaluronic acid injections or oral nonsteroidal anti-inflammatory drugs for knee osteoarthritis: systematic review and meta-analysis of randomized trials. Orthop J Sports Med. 2020;8(1):27. doi:10.1177/2325967119897909

3. Kim I, Kim HA, Seo YI, Song YW, Jeong JY, Kim DH. The prevalence of knee osteoarthritis in elderly community residents in Korea. J Korean Med Sci. 2010;25(2):293. doi:10.3346/jkms.2010.25.2.293

4. Li J, Li YX, Luo LJ, et al. The effectiveness and safety of acupuncture for knee osteoarthritis: an overview of systematic reviews. Medicine (Baltimore). 2019;98(28):e16301. doi:10.1097/MD.0000000000016301

5. Yuan T, Xiong J, Wang X, et al. The effectiveness and safety of moxibustion for treating knee osteoarthritis: a PRISMA compliant systematic review and meta-analysis of randomized controlled trials. Pain Res Manag. 2019;2019:2653792. doi:10.1155/2019/2653792

6. Chen B, Zhan H, Marszalek J, et al. Traditional chinese medications for knee osteoarthritis pain: a meta-analysis of randomized controlled trials. Am J Chin Med. 2016;44(4):677-703. doi:10.1142/S0192415X16500373

7. Gong Z, Liu R, Yu W, Wong TK, Guo Y, Sun Y. Acutherapy for knee osteoarthritis relief in the elderly: a systematic review and meta-analysis. Evid Based Complement Alternat Med. 2019;2019:1868107. doi:10.1155/2019/1868107

8. Park JM, Lee CK, Kim KH, et al. A systematic review and meta-analysis of moxibustion treatment for knee osteoarthritis. $J$ Acupuncture Res. 2020;37(3):137-150. doi:10.13045/jar.2020.00108

9. Kim JH, Yoon YS, Lee WJ, Ko HJ, Kim SJ. A systematic review of herbal medicine treatment for knee osteoarthritis. $J$ Korean Med. 2019;29(4):47-60.

10. Yoh SB, Sul JU, Shin MS. Research trends on the treatment of knee osteoarthritis in Korean medicine. Korean J Acupuncture. 2011;28 (1):139-155.

11. Huang YR, Jin YL, Li N, et al. Effects of acupotomy, electroacupuncture or round-sharp acupuncture needle interventions on expression of Bcl-2, Bax, Caspase-3 proteins of rectus femoris in rabbits with knee ostarthritis. Zhen Ci Yan Jiu. 2014;39(2):100-105, 123.

12. Park HJ, Lee SH, Son DJ, et al. Antiarthritic effect of bee venom: inhibition of inflammation mediator generation by suppression of NF$\kappa \mathrm{B}$ through interaction with the p50 subunit. Arthritis Rheum. 2004;50(11):3504-3515. doi:10.1002/art.20626
13. Chen J, Qiu M, Li Y, et al. Effects of Huatan Tongluo decoction on vascular endothelial growth factor receptor 2 expression in synovial tissues of rats with collagen-induced arthritis. J Tradit Chin Med. 2019;39(2):191-198.

14. Zhang L, Yuan H, Zhang L, Li J, Li H. Effect of acupuncture therapies combined with usual medical care on knee osteoarthritis. $J$ Tradit Chin Med. 2019;39(1):103-110.

15. Kim TH, Kim KH, Kang JW, et al. Moxibustion treatment for knee osteoarthritis: a multi-centre, non-blinded, randomised controlled trial on the effectiveness and safety of the moxibustion treatment versus usual care in knee osteoarthritis patients. PLoS One. 2014;9(7): e101973. doi:10.1371/journal.pone.0101973

16. Song GM, Tian X, Jin YH, et al. Moxibustion is an alternative in treating knee osteoarthritis: the evidence from systematic review and meta-analysis. Medicine. 2016;95(6):e2790. doi:10.1097/ MD.0000000000002790

17. Selfe TK, Taylor AG. Acupuncture and osteoarthritis of the knee: a review of randomized, controlled trials. Fam Community Health. 2008;31(3):247. doi:10.1097/01.FCH.0000324482.78577.0f

18. Long L, Soeken K, Ernst E. Herbal medicines for the treatment of osteoarthritis: a systematic review. Rheumatology. 2001;40 (7):779-793. doi:10.1093/rheumatology/40.7.779

19. Kohn MD, Sassoon AA, Fernando ND. Classifications in brief: kellgren-Lawrence classification of osteoarthritis. Clin Orthopaedics Rel Res. 2016;474(8):1886-1893. doi:10.1007/s11999-016-4732-4

20. Altman R, Asch E, Bloch D, et al. Development of criteria for the classification and reporting of osteoarthritis: classification of osteoarthritis of the knee. Arthritis Rheumatism. 1986;29(8):1039-1049. doi:10.1002/art.1780290816

21. Park HR, Cho SK, Im SG, et al. Treatment patterns of knee osteoarthritis patients in Korea. Korean J Intern Med. 2019;34 (5):1145-1153. doi:10.3904/kjim.2017.304

22. Suzuki Y, Iijima H, Shimoura K, Tsuboyama T, Aoyama T. Patients with early-stage knee osteoarthritis and knee pain have decreased hip abductor muscle strength while descending stairs. Clin Rheumatol. 2019;38(8):2249-2254. doi:10.1007/s10067-019-04523-3

23. Higgins JPT AD, Sterne JAC. Cochrane handbook for systematic reviews of interventions version 5.2.0 (updated June 2017). Cochrane. 2017. Available from www.training.cochrane.org/hand book. Accessed May 6, 2021.

24. Page MJ, McKenzie JE, Bossuyt PM, et al. The PRISMA 2020 statement: an updated guideline for reporting systematic reviews. BMJ. 2021;372:n71. doi:10.1136/bmj.n71
Journal of Pain Research

\section{Publish your work in this journal}

The Journal of Pain Research is an international, peer reviewed, open access, online journal that welcomes laboratory and clinical findings in the fields of pain research and the prevention and management of pain. Original research, reviews, symposium reports, hypothesis formation and commentaries are all considered for publication. The manuscript

Submit your manuscript here: https://www.dovepress.com/journal-of-pain-research-journal management system is completely online and includes a very quick and fair peer-review system, which is all easy to use. Visit http:// www.dovepress.com/testimonials.php to read real quotes from published authors. 